\title{
Degree of polarization of type-II unpolarized light
}

\author{
Alfredo Luis* \\ Departamento de Óptica, Facultad de Ciencias Físicas, Universidad Complutense, 28040 Madrid, Spain
}

(Received 14 January 2007; published 7 May 2007)

\begin{abstract}
We address a quantitative determination of the degree of polarization of type-II unpolarized light via the computation of the distance between the polarization distribution and the uniform distribution associated with fully unpolarized light (i.e., type-I unpolarized light or natural light). We determine the maximum degree of polarization for type-II unpolarized light and the states reaching it. We show that the degree of polarization can be arbitrarily large, approaching complete polarization for increasing mean photon numbers.
\end{abstract}

DOI: 10.1103/PhysRevA.75.053806

PACS number(s): 42.50.Dv, 03.65.Ca, 42.25.Ja

\section{INTRODUCTION}

The intuitive idea of unpolarized light can be formalized by postulating specific invariance properties under transformations. Two classes of unpolarized light have been defined, types I and II, depending on the set of symmetries actually satisfied [1-3]. [2].

Type-II unpolarized light satisfies the following properties

(i) Rotational invariance with respect to the propagation direction. These rotations can be implemented for example via optical activity or Faraday effect.

(ii) Symmetry with respect to left- and right-handed circular polarization. This interchange can be achieved by the action of half-wave plates.

Type-I unpolarized light (or natural light) corresponds to the fulfillment, in addition to (i) and (ii), of a further property [1]:

(iii) Invariance with respect to phase changes between linearly polarized components. These phase changes can be implemented by standard phase plates.

For both types I and II the Stokes parameters vanish, so that they would be both unpolarized according to the classic criterion, where the degree of polarization is measured in terms of the length of the three-dimensional vector of Stokes parameters. This definition is suitable for the thermal-chaotic fields produced by most light sources, since in such a case vanishing Stokes parameters are equivalent to natural light. However, the Stokes parameters are insufficient to properly assess the amount of polarization for other field states.

In recent years some measures of the degree of polarization have been proposed to solve this difficulty, in both the quantum and classical domains [4-6]. It can be seen that the entropic measures $[4,5]$ are well behaved in the classical limit and provide essentially equivalent results [5].

In this work we apply the approach in Ref. [5], which is recalled in Sec. II, measuring the degree of polarization as the distance between the polarization distribution and the uniform distribution associated with type-I unpolarized light. We also recall in Sec. II the definition and main properties of type-II unpolarized light. In Sec. III we examine the amount

\footnotetext{
*Electronic address: alluis@ fis.ucm.es; URL: http://www.ucm.es/ info/gioq
}

of polarization conveyed by type-II unpolarized light, showing that it can be as close as desired to complete polarization by increasing mean photon numbers. Finally, in Sec. IV we determine the maximum degree of polarization for type-II unpolarized light with a fixed number of photons and the states reaching it.

\section{PRELIMINARY MATERIAL}

This section recalls the main concepts and definitions required for the analysis developed in Secs. III and IV.

\section{A. Degree of polarization}

Throughout we will assume a monochromatic field described by two annihilation operators $a_{+}$and $a_{-}$representing the complex amplitude of circularly polarized modes. Polarization properties are suitably described by the Stokes operators

$$
\begin{gathered}
S_{0}=a_{+}^{\dagger} a_{+}+a_{-}^{\dagger} a_{-}, \quad S_{x}=a_{+}^{\dagger} a_{-}+a_{-}^{\dagger} a_{+}, \\
S_{y}=i\left(a_{-}^{\dagger} a_{+}-a_{+}^{\dagger} a_{-}\right), \quad S_{z}=a_{+}^{\dagger} a_{+}-a_{-}^{\dagger} a_{-} .
\end{gathered}
$$

Their mean values are the Stokes parameters $\left\langle S_{0}\right\rangle$ and $\langle\mathbf{S}\rangle$. Complete statistical information about polarization is provided by the probability distribution on the surface of the Poincaré sphere. In the quantum domain this role can be suitably played by the $\mathrm{SU}(2) Q$ function [5]

$$
Q(\Omega)=\sum_{n=0}^{\infty} \frac{n+1}{4 \pi}\langle n, \Omega|\rho| n, \Omega\rangle,
$$

where $\rho$ is the density matrix for the two-mode field state, $|n, \Omega\rangle$ are the $\mathrm{SU}(2)$-coherent states [7],

$$
|n, \Omega\rangle=\sum_{m=0}^{n}\left(\begin{array}{c}
n \\
m
\end{array}\right)^{1 / 2}\left(\cos \frac{\theta}{2}\right)^{m}\left(\sin \frac{\theta}{2}\right)^{n-m} e^{-i m \phi}|m, n-m\rangle,
$$

and $|m, n-m\rangle=|m\rangle_{+}|n-m\rangle_{-}$denote the product of photon number states in the corresponding mode. The points on the Poincaré sphere will be mostly represented as $\Omega=(\theta, \phi)$, where $\theta$ and $\phi$ are the polar and azimuthal angles, respectively, with $\pi \geqslant \theta \geqslant 0,2 \pi \geqslant \phi \geqslant 0$. 
It is worth noting that in Eq. (2.2) the matrix elements of $\rho$ connecting subspaces of different total photon number $n$ do not contribute. This is consistent with the fact that in classical optics polarization and intensity are independent concepts-i.e., the form of the ellipse described by the electric vector (polarization) and the size of the ellipse (intensity). This does not exclude that for particular cases, such as Gaussian field states, some definite relations between them may arise [8] because of the small number of parameters characterizing these states.

The degree of polarization of a given field state $\rho$ can be measured by the distance between its $Q$ function and the uniform distribution $Q(\Omega)=1 /(4 \pi)$ associated with type-I unpolarized light [1]

$$
D=4 \pi \int d \Omega\left[Q(\Omega)-\frac{1}{4 \pi}\right]^{2}=4 \pi \int d \Omega Q^{2}(\Omega)-1,
$$

where $d \Omega=\sin \theta d \theta d \phi$ is the differential of the solid angle. It is worth noting that $D$ is not bounded from above. If a normalized measure were required, we may consider, for example,

$$
P=\frac{D}{D+1}=1-\frac{1}{4 \pi \mathcal{D}},
$$

where

$$
\mathcal{D}=\int d \Omega Q^{2}(\Omega)
$$

and $1 / \mathcal{D}$ is a measure of the effective area of the Poincare sphere occupied by $Q(\Omega)[5,9]$. This and similar definitions have been already used as measures of localization, uncertainty, and information in different contexts [9].

The maximum $\mathcal{D}$ for fixed $n$ is reached by the $\mathrm{SU}(2)$ coherent states (2.3) with [5]

$$
\mathcal{D}=\frac{(n+1)^{2}}{4 \pi(2 n+1)} \simeq \frac{n}{8 \pi},
$$

where the approximation holds for $n \gg 1$. For example, for the $\mathrm{SU}(2)$-coherent state $|n\rangle_{+}|0\rangle_{-}$located at the north pole $\theta$ $=0$, the polarization distribution is

$$
Q(\Omega)=\frac{n+1}{4 \pi}\left(\cos \frac{\theta}{2}\right)^{2 n} .
$$

When $n \gg 1$ this distribution is sharply peaked around $\theta=0$, so that the following approximation holds:

$$
Q(\Omega) \simeq \frac{n}{4 \pi}\left(1-\frac{\theta^{2}}{8}\right)^{2 n} \simeq \frac{n}{4 \pi} \exp \left(-n \theta^{2} / 4\right),
$$

which is consistent with the scaling of $\mathcal{D}$ in Eq. (2.7) for $n$ $\gg 1$.

\section{B. Type-II unpolarized light}

The Poincaré sphere provides a nice and useful picture of the transformation properties involved in the idea of type-II unpolarized light. Taking into account that the poles represent circular polarization, the rotation around the axis of propagation corresponds to the rotation on the sphere around the axis passing through the poles, $\phi \rightarrow \phi+\phi_{0}$ for arbitrary $\phi_{0}$, which are generated by $S_{z}$. Therefore, condition (i) implies that $Q(\Omega)$ does not depend on $\phi$, and, in particular $\left\langle S_{x}\right\rangle=\left\langle S_{y}\right\rangle=0$. In Hilbert space, condition (i) implies that $\left[S_{z}, \rho\right]=0$, so that the density matrix in the number basis reads

$$
\rho=\sum_{n=0}^{\infty} \sum_{m=0}^{n} p_{n, m}|m, n-m\rangle\langle m, n-m|+(\text { crossed terms }),
$$

where "crossed terms" refer to matrix elements between subspaces of different total photon number $n \neq n^{\prime}$, which according to Eqs. (2.2) and (2.3) do not contribute to $Q(\Omega)$.

On the other hand, the symmetry with respect to circular polarization means invariance with respect to the transformation $S_{z} \rightarrow-S_{z}$-i.e., $\theta \rightarrow \pi-\theta$-so that $Q(\Omega)$ does not contain odd powers of $\cos \theta$, and, in particular, $\left\langle S_{z}\right\rangle=0$. In Hilbert space, the condition (ii) implies that $p_{n, m}=p_{n, n-m}$ in Eq. (2.10).

The vanishing of the three Stokes parameters as a consequence of (i) and (ii) qualifies these states as unpolarized light according to the classic criterion.

\section{DEGREE OF POLARIZATION OF TYPE-II UNPOLARIZED LIGHT}

In this section we measure the degree of polarization for meaningful and practical examples of type-II unpolarized light by the computation of $\mathcal{D}$ in Eq. (2.6).

\section{A. Classically maximally correlated states}

Let us consider the following states $\rho_{n}$ within a definite subspace of fixed total number of photons $n$ expressed in the number basis as

$$
\rho_{n}=\frac{1}{2}(|n, 0\rangle\langle n, 0|+| 0, n\rangle\langle 0, n|),
$$

which are clearly of the form (2.10) with $p_{n, m}=p_{n, n-m}$. These states are incoherent superpositions of two SU(2)-coherent states located at the poles of the Poincaré sphere with

$$
Q(\Omega)=\frac{n+1}{8 \pi}\left[\left(\cos \frac{\theta}{2}\right)^{2 n}+\left(\sin \frac{\theta}{2}\right)^{2 n}\right],
$$

leading to

$$
\mathcal{D}=\frac{1}{8 \pi} \frac{(n+1)^{2}}{2 n+1}\left[1+\frac{n !^{2}}{(2 n) !}\right] \simeq \frac{n}{16 \pi},
$$

where the approximation holds for $n \gg 1$.

We can see that the degree of polarization can be actually very large, with $\mathcal{D} \rightarrow \infty$ and $P \rightarrow 1$ when $n \rightarrow \infty$. Actually, Eq. (3.3) is just half of the absolute maximum (2.7). This result is consistent since Eq. (3.2) corresponds to two coherent states 
located at the poles, so that when $n \gg 1$ they do not overlap and occupy twice the area of a single coherent state. In Sec. IV we show that Eq. (3.3) is the maximum $\mathcal{D}$ for type-II unpolarized light with definite total photon number $n$.

The states $\rho_{n}$ are difficult to generate, since they are essentially number states. Nevertheless, there are feasible states with equivalent polarization properties such as

$$
\begin{aligned}
\rho_{\alpha} & =\frac{1}{2}(|\alpha, 0\rangle\langle\alpha, 0|+| 0, \alpha\rangle\langle 0, \alpha|) \\
& =e^{-\bar{n}} \sum_{n=0}^{\infty} \frac{\bar{n}^{n}}{n !} \rho_{n}+\text { (crossed terms), }
\end{aligned}
$$

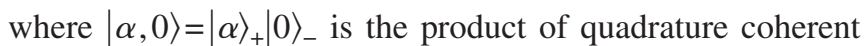
states $a_{ \pm}\left|\alpha_{ \pm}\right\rangle_{ \pm}=\alpha_{ \pm}\left|\alpha_{ \pm}\right\rangle_{ \pm}$and $\bar{n}=|\alpha|^{2}$. This state corresponds to the random generation of right- and left-handed circularly polarized light with equal probabilities.

The exact $Q(\Omega)$ corresponding to $\rho_{\alpha}$ is

$$
\begin{aligned}
Q(\Omega)= & \frac{1}{8 \pi}\left[\left(\bar{n} \cos ^{2} \frac{\theta}{2}+1\right) \exp \left(-\bar{n} \sin ^{2} \frac{\theta}{2}\right)\right. \\
& \left.+\left(\bar{n} \sin ^{2} \frac{\theta}{2}+1\right) \exp \left(-\bar{n} \cos ^{2} \frac{\theta}{2}\right)\right],
\end{aligned}
$$

leading to

$$
\mathcal{D}=\frac{1}{16 \pi}\left[1+\bar{n}+\frac{1}{2 \bar{n}}-\frac{e^{-2 \bar{n}}}{2 \bar{n}}+e^{-\bar{n}}\left(\frac{\bar{n}^{2}}{3}+2 \bar{n}+2\right)\right] .
$$

These expressions can be suitably approximated in the limit $\bar{n} \gg 1$ as $\cos (\theta / 2) \simeq 1$ and $\sin (\theta / 2) \simeq \theta / 2$ for the first term in Eq. (3.5) and $\cos (\theta / 2) \simeq-(\theta-\pi) / 2$ and $\sin (\theta / 2) \simeq 1$ for the second, leading to

$$
Q(\Omega) \simeq \frac{\bar{n}}{8 \pi}\left\{\exp \left(-\bar{n} \theta^{2} / 4\right)+\exp \left[-\bar{n}(\theta-\pi)^{2} / 4\right]\right\},
$$

which is fully compatible with the $\bar{n} \gg 1$ limit of Eq. (3.6):

$$
\mathcal{D} \simeq \frac{\bar{n}}{16 \pi} .
$$

\section{B. Phase-averaged equatorial-coherent states}

Conditions (i) and (ii) are also satisfied by the phaseaveraged equatorial $\mathrm{SU}(2)$-coherent states

$$
\rho_{n}=\frac{1}{2 \pi} \int d \phi\left|n, \theta=\frac{\pi}{2}, \phi\right\rangle\left\langle n, \theta=\frac{\pi}{2}, \phi\right|,
$$

which are the incoherent superposition of all the SU(2)coherent states $|n, \theta, \phi\rangle$ located at the equator of the Poincaré sphere $\theta=\pi / 2$. In the number basis we have

$$
\rho_{n}=\frac{1}{2^{n}} \sum_{m=0}^{n}\left(\begin{array}{l}
n \\
m
\end{array}\right)|m, n-m\rangle\langle m, n-m|,
$$

which is clearly of the form (2.10) with $p_{n, m}=p_{n, n-m}$. The corresponding polarization distribution is

$$
Q(\Omega)=\frac{n+1}{4 \pi 2^{n}} \sum_{m=0}^{n}\left(\begin{array}{l}
n \\
m
\end{array}\right)^{2}\left(\cos \frac{\theta}{2}\right)^{2 m}\left(\sin \frac{\theta}{2}\right)^{2 n-2 m} .
$$

We have not been able to derive an exact closed expression for $\mathcal{D}$. A Gaussian approximation of $Q(\Omega)$ valid for $n$ $\gg 1$ can be developed as follows. For $n \gg 1$ the binomial coefficient tends to be Gaussian,

$$
\left(\begin{array}{l}
n \\
m
\end{array}\right) \simeq \frac{2^{n+1}}{\sqrt{2 \pi n}} \exp \left[-\frac{2}{n}(m-n / 2)^{2}\right],
$$

and the $m$ sum can be replaced by an integral $\Sigma_{m} \rightarrow \int d m$, leading to

$$
Q(\Omega) \simeq \frac{\sqrt{n}}{4 \pi \sqrt{\pi}} \sin ^{n} \theta \exp \left[\frac{n}{16} \ln ^{2}\left(\tan ^{2} \frac{\theta}{2}\right)\right] .
$$

The distribution (3.11) is sharply located around the equator, so we can further simplify Eq. (3.13) by approximating the harmonic functions around $\theta=\pi / 2$, leading finally to

$$
Q(\Omega) \simeq \frac{\sqrt{n}}{4 \pi \sqrt{\pi}} \exp \left[-\frac{n}{4}(\theta-\pi / 2)^{2}\right],
$$

which allows us to compute the leading term of $\mathcal{D}$ as a function of $n$ as

$$
\mathcal{D} \simeq \sqrt{\frac{n}{32 \pi^{3}}} .
$$

As in the preceding example the states (3.9) are difficult to generate. Their feasible counterparts are the products $\rho_{+} \rho_{-}$ of randomly phased mixtures of quadrature-coherent states, which are the output of a laser well above threshold [10],

$$
\rho_{j}=\frac{1}{2 \pi} \int d \phi\left|e^{i \phi} \sqrt{\bar{n} / 2}\right\rangle_{j j}\left\langle e^{i \phi} \sqrt{\bar{n} / 2}\right|,
$$

where $\left|e^{i \phi} \sqrt{\bar{n} / 2}\right\rangle_{j}$ are quadrature-coherent states in modes $j$ $= \pm$ with complex amplitudes $\alpha=e^{i \phi \sqrt{n} / 2}$. The product $\rho_{+} \rho_{-}$ can be expressed as

$$
\rho_{+} \rho_{-}=e^{-\bar{n}} \sum_{n=0}^{\infty} \frac{\bar{n}^{n}}{n !} \rho_{n},
$$

where $\rho_{n}$ are in Eq. (3.9). For large enough $\bar{n} \gg 1$ the Poisson distribution in Eq. (3.17) tends to be sharply peaked around $n \simeq \bar{n}$ so that the approximations (3.14) and (3.15) will hold for the state (3.17) replacing $n$ by $\bar{n}$.

We can appreciate that also in this case we have increasing degree of polarization $\mathcal{D} \rightarrow \infty$ and $P \rightarrow 1$ for increasing photon numbers $n \rightarrow \infty$, although at a less efficient rate than the preceding example. 


\section{Twin photon number states}

Another known field state satisfying conditions (i) and (ii) is the product of number states with an equal number of photons in each mode, $|n, n\rangle=|n\rangle_{+}|n\rangle_{-}$. The polarization properties of these states have been already considered in Ref. [5], so we just reproduce here the results for the sake of completeness. The exact polarization distribution and a suitable Gaussian approximation valid for $n \gg 1$ are

$$
Q(\Omega)=\frac{2 n+1}{4 \pi 2^{2 n}}\left(\begin{array}{c}
2 n \\
n
\end{array}\right) \sin ^{2 n} \theta \simeq \frac{1}{2 \pi} \sqrt{\frac{n}{\pi}} e^{-n(\theta-\pi / 2)^{2}},
$$

leading to

$$
\mathcal{D}=\frac{(2 n+1)^{2}}{4 \pi(4 n+1)}\left(\begin{array}{c}
2 n \\
n
\end{array}\right)^{2}\left(\begin{array}{c}
4 n \\
2 n
\end{array}\right)^{-1} \simeq \sqrt{\frac{n}{8 \pi^{3}}} .
$$

The feasible practical counterpart of this state is the twomode squeezed vacuum state

$$
|\xi\rangle=\frac{1}{\sqrt{1+\bar{n}}} \sum_{n=0}^{\infty}\left(\frac{\bar{n}}{1+\bar{n}}\right)^{n / 2}|n, n\rangle,
$$

where $\bar{n}$ represents the mean number of photons, leading to

$$
Q(\Omega)=\frac{1}{1+\bar{n}} \sum_{n=0}^{\infty}\left(\frac{\bar{n}}{1+\bar{n}}\right)^{n} \frac{2 n+1}{4 \pi 2^{2 n}}\left(\begin{array}{c}
2 n \\
n
\end{array}\right) \sin ^{2 n} \theta,
$$

which can be suitably approximated for $\bar{n} \gg 1$ by replacing the $n$ sum by an integral and using the approximation in Eq. (3.18), so that

$$
Q(\Omega) \simeq \frac{\sqrt{\bar{n}}}{4 \pi} \frac{1}{\left[1+\bar{n}(\theta-\pi / 2)^{2}\right]^{3 / 2}}
$$

and

$$
\mathcal{D} \simeq \frac{3}{64} \sqrt{\bar{n}}
$$

The conclusion is that this example also reproduces the preceding patterns since $\mathcal{D} \rightarrow \infty$ and $P \rightarrow 1$ for $n, \bar{n} \rightarrow \infty$.

\section{MAXIMUM DEGREE OF POLARIZATION FOR TYPE-II UNPOLARIZED LIGHT}

In this section we determine the maximum $\mathcal{D}$ allowed for type-II unpolarized light for fixed total photon number $n$. The general form (2.10) for fixed $n$ implies that

$$
Q(\Omega)=\sum_{m=0}^{[n / 2]} \tilde{p}_{n, m} \widetilde{Q}_{n, m}(\Omega),
$$

where $[n / 2]$ denotes the greatest integer less than or equal to $n / 2, \tilde{p}_{n, m}=2 p_{n, m}$ for $m \neq n / 2$, with $\tilde{p}_{n, n / 2}=p_{n, n / 2}$, and

$$
\widetilde{Q}_{n, m}(\Omega)=\frac{1}{2}\left[Q_{n, m}(\Omega)+Q_{n, n-m}(\Omega)\right],
$$

where

$$
\begin{aligned}
Q_{n, m}(\Omega) & =\frac{n+1}{4 \pi}|\langle n, \theta, \phi \mid m, n-m\rangle|^{2} \\
& =\frac{n+1}{4 \pi}\left(\begin{array}{l}
n \\
m
\end{array}\right)\left(\cos \frac{\theta}{2}\right)^{2 m}\left(\sin \frac{\theta}{2}\right)^{2(n-m)} .
\end{aligned}
$$

Therefore

$$
\mathcal{D}=\sum_{m, m^{\prime}=0}^{[n / 2]} \tilde{p}_{n, m} \tilde{p}_{n, m^{\prime}} \int d \Omega \tilde{Q}_{n, m}(\Omega) \tilde{Q}_{n, m^{\prime}}(\Omega) .
$$

Using the Cauchy-Swartz inequality we get

$$
\mathcal{D} \leqslant \sum_{m, m^{\prime}=0}^{[n / 2]} \tilde{p}_{n, m} \tilde{p}_{n, m^{\prime}} \sqrt{\int d \Omega \widetilde{Q}_{n, m}^{2}(\Omega) \int d \Omega^{\prime} \tilde{Q}_{n, m^{\prime}}^{2}\left(\Omega^{\prime}\right)} .
$$

The right-hand side of this equation is bounded from above by the maximum of $\int d \Omega \widetilde{Q}_{n, m}^{2}(\Omega)$ when $m$ is varied, where

$$
\int d \Omega \widetilde{Q}_{n, m}^{2}(\Omega)=\frac{(n+1)^{2}}{8 \pi(2 n+1)}\left(\begin{array}{l}
n \\
m
\end{array}\right)^{2}\left[\left(\begin{array}{c}
2 n \\
2 m
\end{array}\right)^{-1}+\frac{n !^{2}}{(2 n) !}\right] .
$$

We have checked numerically this expression for all values of $n$ up to $n=10^{5}$, finding that, with the only exception of $n=2,4$, it takes the maximum when $m=0$, which corresponds to the maximally correlated states (3.1), so that

$$
\mathcal{D} \leqslant \frac{(n+1)^{2}}{8 \pi(2 n+1)}\left[1+\frac{n !^{2}}{(2 n) !}\right] \text {. }
$$

For $n=2,4$ the maximum occurs for $m=n / 2$.

\section{CONCLUSIONS}

We have shown that type-II unpolarized light can attain an arbitrarily large degree of polarization when this is measured as the distance between the polarization distribution and the uniform distribution associated with fully unpolarized light (type I). This is in sharp contradiction with its name and with the vanishing of the classic degree of polarization.

The key point for this result is to measure the amount of polarization in terms of statistical evaluations of polarization beyond the first moments of the Stokes operators (Stokes parameters). The classic approach is appropriate for the thermal-chaotic light produced by most classical light sources since the first moments determine the whole distribution, but becomes incomplete when applied to other field states, especially in the quantum domain.

We have applied this approach to somewhat sophisticated quantum states in Eqs. (3.1), (3.9), and (3.20), as well as to the standard classical-like states in Eqs. (3.4) and (3.17), which are incoherent superpositions of quadrature-coherent states. Thus it seems that there is no definite relation between the degree of polarization of type-II unpolarized states and their potential quantum properties. As a matter of fact, 
type-II unpolarized light has been also examined from the perspective of classical optics [3].

It is worth pointing out that the states (3.4) and (3.17) are classical according to most measures of quantum behavior, including those based on the positivity of $s$-ordered quadrature distributions $[11,12]$. However, the distinction between quantum and classical light can be rather subtle, in particular because of the existence of phase-space representations of quantum states different from $s$-ordered quadrature distributions [13]. Concerning polarization, we have shown in Ref.
[14] that quadrature-coherent states may display nonclassical polarization properties.

\section{ACKNOWLEDGMENTS}

A.L. acknowledges support from Project No. FIS200401814 of the Spanish Dirección General de Investigación del Ministerio de Educación y Ciencia and Project No. PR1-A/ 07-15378 of the Universidad Complutense.
[1] G. S. Agarwal, Lett. Nuovo Cimento Soc. Ital. Fis. 1, 53 (1971); H. Prakash and N. Chandra, Phys. Rev. A 4, 796 (1971).

[2] G. S. Agarwal, J. Lehner, and H. Paul, Opt. Commun. 129, 369 (1996); J. Lehner, U. Leonhardt, and H. Paul, Phys. Rev. A 53, 2727 (1996); J. Lehner, H. Paul, and G. S. Agarwal, Opt. Commun. 139, 262 (1997).

[3] J. Ellis and A. Dogariu, J. Opt. Soc. Am. A 21, 988 (2004); 22, 491 (2005).

[4] A. Picozzi, Opt. Lett. 29, 1653 (2004); P. Réfrégier, F. Goudail, P. Chavel, and A. Friberg, J. Opt. Soc. Am. A 21, 2124 (2004); P. Réfrégier, Opt. Lett. 30, 1090 (2005); P. Réfrégier, M. Roche, and F. Goudail, J. Opt. Soc. Am. A 23, 124 (2006); P. Réfrégier and F. Goudail, ibid. 23, 671 (2006); P. Réfrégier and J. Morio, ibid. 23, 3036 (2006).

[5] A. Luis, Phys. Rev. A 66, 013806 (2002); 71, 063815 (2005); Opt. Commun. 273, 173 (2007).

[6] A. B. Klimov, L. L. Sánchez-Soto, E. C. Yustas, J. Söderholm, and G. Björk, Phys. Rev. A 72, 033813 (2005); A. Sehat, J. Söderholm, G. Björk, P. Espinoza, A. B. Klimov, and L. L. Sánchez-Soto, ibid. 71, 033818 (2005).

[7] F. T. Arecchi, E. Courtens, R. Gilmore, and H. Thomas, Phys. Rev. A 6, 2211 (1972).

[8] E. Wolf, Proc. Phys. Soc. London 76, 424 (1960); T. Setälä, K. Lindfors, M. Kaivola, J. Tervo, and A. T. Friberg, Opt. Lett. 29, 2587 (2004).

[9] A. Wehrl, Rev. Mod. Phys. 50, 221 (1978); E. J. Heller, Phys. Rev. A 35, 1360 (1987); H. Maassen and J. B. M. Uffink, Phys. Rev. Lett. 60, 1103 (1988); U. Larsen, J. Phys. A 23,
1041 (1990); A. Wehrl, Rep. Math. Phys. 30, 119 (1991); I. Bialynicki-Birula, M. Freyberger, and W. Schelich, Phys. Scr. T48, 113 (1993); A. Anderson and J. J. Halliwell, Phys. Rev. D 48, 2753 (1993); A. Lukš and V. Peřinová, Quantum Opt. 6, 125 (1994); B. Mirbach and H. J. Korsch, Ann. Phys. (N.Y.) 265, 80 (1998); Č. Brukner and A. Zeilinger, Phys. Rev. Lett. 83, 3354 (1999); Phys. Rev. A 63, 022113 (2001); M. J. W. Hall, ibid. 59, 2602 (1999); S. Gnutzmann and K. Życzkowski, J. Phys. A 34, 10123 (2001); A. Luis, Phys. Rev. A 67, 032108 (2003); Opt. Lett. 31, 3644 (2006).

[10] L. Mandel and E. Wolf, Optical Coherence and Quantum Optics (Cambridge University Press, New York, 1995).

[11] M. Hillery, Phys. Rev. A 35, 725 (1987); P. Marian, T. A. Marian, and H. Scutaru, Phys. Rev. Lett. 88, 153601 (2002); Phys. Rev. A 69, 022104 (2004).

[12] C. T. Lee, Phys. Rev. A 44, R2775 (1991); 52, 3374 (1995); N. Lütkenhaus and S. M. Barnett, ibid. 51, 3340 (1995); A. F. de Lima and B. Baseia, ibid. 54, 4589 (1996); J. Janszky, M. G. Kim, and M. S. Kim, ibid. 53, 502 (1996); M. G. Benedict and A. Czirják, ibid. 60, 4034 (1999); A. Kenfack and K. Zyczkowski, J. Opt. B: Quantum Semiclassical Opt. 6, 396 (2004).

[13] J. A. Vaccaro, Opt. Commun. 113, 421 (1995); Phys. Rev. A 52, 3474 (1995); J. A. Vaccaro and D. T. Pegg, ibid. 41, 5156 (1990); L. M. Johansen, Phys. Lett. A 329, 184 (2004); J. Opt. B: Quantum Semiclassical Opt. 6, L21 (2004); L. M. Johansen and A. Luis, Phys. Rev. A 70, 052115 (2004).

[14] A. Luis, Phys. Rev. A 73, 063806 (2006). 